Artículos científicos

\title{
La movilidad académica en la escuela normal: una estrategia para la consolidación institucional
}

\author{
Academic mobility as an strategy for institutional consolidation at the Normal \\ school
}

\begin{abstract}
Mobilidade acadêmica na escola normal: uma estratégia de consolidação institucional
\end{abstract}

\section{Román Castro Miranda \\ Benemérita Escuela Normal Veracruzana Enrique C. Rébsamen, México romancastrobenv@gmail.com https://orcid.org/0000-0002-8905-7286}

Héctor Gabriel Rangel Ramírez Universidad Veracruzana, Instituto de Investigaciones y Estudios Superiores Económicos y Sociales, México hrangel@uv.mx https://orcid.org/0000-0002-1507-4593

\section{Resumen}

La internacionalización y su componente de movilidad académica representan una estrategia prioritaria en la agenda de desarrollo de las instituciones de educación superior públicas y privadas de todo el mundo. Las escuelas normales de México han vivido reformas importantes que han reivindicado su papel como instituciones clave en la formación de profesionales de la educación en nuestro país, aunque en el ámbito de la internacionalización, aún presentan más retos que realidades. El presente artículo es resultado de una investigación documental y descriptiva que pretende generar un diálogo intersaberes sobre la perspectiva que las escuelas normales en México tienen hacia la implementación de la movilidad académica en su estructura institucional, resaltando la importancia que esta enmarca en el proceso de la formación integral 


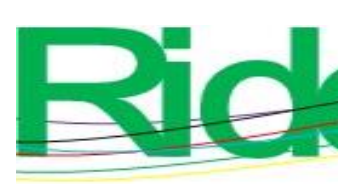

Revista Iberoamericana para la Investigación y el Desarrollo Educativo ISSN 2007 - 7467

y en donde compartimos algunos indicadores de desempeño que existen actualmente a nivel nacional e internacional respecto a las transformaciones organizacionales, curriculares, administrativas y financieras que se deben realizar para implementar exitosamente la dimensión internacional e intercultural en las funciones sustantivas de dichas instituciones.

Palabras clave: internacionalización, formación integral, movilidad académica, modelo educativo, planeación.

\section{Abstract}

Internationalization and its component academic mobility represents a core strategy in the development agenda of both public and private higher education institutions around the world. Teacher Training schools in Mexico, best known as "escuelas normales", have experienced important reforms that have redeemed its role as key institutions in the formation of education professionals in our country, however, in the field of internationalization, they still have more challenges than realities. This document results from a detailed descriptive and documentary research which aims to unfold an interdisciplinary dialogue on the escuelas normales' perspective towards the implementation of the academic mobility in their institutional structure, highlighting its importance within the integral formation process, sharing the main performance indicators at a national level, and identifying the organizational, curricular, administrative and financial transformations needed to successfully implement de international and intercultural dimension in the core functions of such institutions.

Keywords: Internationalization, integral formation, academic mobility, educational model, planning.

\section{Resumo}

A internacionalização e seu componente de mobilidade acadêmica representam uma estratégia prioritária na agenda de desenvolvimento das instituições de ensino superior públicas e privadas em todo o mundo. As escolas normais no México passaram por reformas importantes que têm reivindicado seu papel como instituições fundamentais na formação de profissionais da educação em nosso país, embora no campo da internacionalização ainda apresentem mais desafios do que realidades. Este artigo é o resultado de uma pesquisa documental e descritiva que visa gerar um diálogo interserviços sobre a perspectiva que as escolas normais do México têm em relação à 


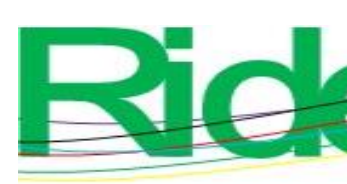

Revista Iberoamericana para la

Investigación y el Desarrollo Educativo

ISSN 2007 - 7467

implementação da mobilidade acadêmica em sua estrutura institucional, destacando a importância que esta enquadra no processo de formação integral e onde partilhamos alguns indicadores de desempenho que existem atualmente a nível nacional e internacional sobre as transformações organizacionais, curriculares, administrativas e financeiras que se devem realizar para concretizar com sucesso a dimensão internacional e intercultural nas funções substantivas das referidas instituições.

Palavras-chave: internacionalização, formação integral, mobilidade acadêmica, modelo educacional, planejamento.

Fecha Recepción: Junio 2020

Fecha Aceptación: Octubre 2020

\section{Introducción}

La movilidad académica en México ha tomado apenas en las últimas dos décadas un significado relevante en términos del desarrollo de competencias profesionales. En el contexto universitario, desde sus referentes curriculares, es una pieza clave para la formación del estudiantado, así como en la formación y actualización del propio profesorado.

Las escuelas normales en nuestro país, aun cuando son referentes obligados en la formación de docentes, son prácticamente ajenas a la adopción de acciones formales de movilidad académica, ya que apenas en los últimos siete años se han presentado los primeros esfuerzos, los cuales aún son poco reconocidos y apoyados por autoridades y el propio profesorado que los atiende.

La globalización del conocimiento demanda al sector educativo, y en especial a las escuelas formadoras de docentes, transformar su visión institucional para abandonar zonas de confort y dejar modelos académicos que conducen a procesos unidireccionales, cerrados a la diversidad, acotados a la pertinencia local y ubicados en contextos tradicionales.

En este texto, por ende, se pretende analizar la importancia que tiene la implementación de políticas y acciones estratégicas en materia de internacionalización de la educación superior, particularmente la participación de las escuelas normales en esquemas de movilidad académica.

En el primer apartado se describe el contexto actual de las escuelas normales con respecto a la globalización y a los procesos de internacionalización que se están suscitando en la educación superior; posteriormente, se argumenta por qué la movilidad como experiencia vivencial, formadora y con objetivos a nivel curricular tiene alto impacto en el proceso de formación integral del estudiantado normalista. 


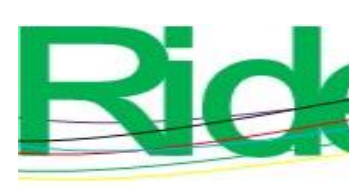

Revista Iberoamericana para la Investigación y el Desarrollo Educativo ISSN $2007-7467$

Así mismo, se presentan algunas coordenadas cuantitativas sobre los flujos de movilidad que se han podido registrar a nivel mundial y particularmente en nuestro país, de tal forma que se pueda proyectar una mirada que identifique el avance que han tenido otras instituciones de educación superior en México y conocer algunas tendencias relacionadas con los destinos preferidos para realizar estancias, esquemas de participación, movilidad entrante versus saliente y las instituciones que lideran estas actividades a nivel nacional.

Finalmente, se analizan escenarios factibles para la implementación de la movilidad en las escuelas normales, haciendo hincapié en el último apartado sobre la serie de transformaciones económicas, académicas, culturales y de gestión, que podrían realizar las escuelas normales para asegurar un camino viable, sostenido y sobre todo estratégico, hacia la implementación de la movilidad académica, como un mecanismo para favorecer el desarrollo de sus funciones sustantivas con calidad y pertinencia global.

\section{El contexto de las escuelas normales públicas ante la internacionalización}

El magisterio y la serie de procesos que emanan de su estructura representan la base de la formación de los profesionales en México. Las escuelas normales, a pesar de los constantes retos y críticas que han recibido a lo largo de su historia, deberán seguir siendo los espacios por excelencia para configurar y operar la formación inicial. La profesión del ser docente se ha transformado a través del tiempo según las circunstancias sociales, económicas e incluso políticas de nuestro país; particularmente, hoy se sigue debatiendo el papel y la pertinencia que la formación docente ocupa en la agenda educativa nacional. En este sentido, la Organización de las Naciones Unidas para la Educación, la Ciencia y la Cultura (Unesco) (2010) afirma que el profesorado constituye el recurso más importante de la educación en todos los países. Por eso, desde la enseñanza preescolar y a lo largo de toda la enseñanza primaria y secundaria, la presencia del docente calificado y motivado es esencial para un aprendizaje eficaz (Unesco, 2010).

Si bien se reconoce el papel del maestro a nivel mundial, el desempeño, la formación y los resultados de enseñanza en nuestro país parecen mostrar que aún son más los retos que los alcances consolidados. Muchos de los cuestionamientos que se han hecho al magisterio han emanado de las evaluaciones estandarizadas, cuyos resultados han dejado ver que el nivel de aprendizaje del estudiantado de todos los niveles ha estado por debajo del promedio nacional, y por mucho a nivel internacional. Una de las contribuciones más importantes en educación básica 


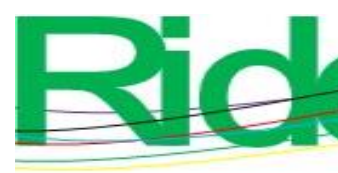

Revista Iberoamericana para la Investigación y el Desarrollo Educativo ISSN 2007 - 7467

y con un gran peso en la medición de los avances en materia de aprovechamiento escolar es el Programa para la Evaluación Internacional de los Estudiantes (PISA, por sus siglas en inglés). La Organización para la Cooperación y el Desarrollo Económico (OCDE) es el organismo que coordina este programa de evaluación, a través del cual se lleva a cabo un estudio comparativo entre los sistemas educativos de los países miembros. La evaluación realizada por PISA está basada en el enfoque de competencias, el cual ha tenido un fuerte impacto en el rediseño de planes y programas educativos en los diferentes niveles. Por mencionar un ejemplo del impacto de este programa - después de la primera administración de estas evaluaciones en educación superior - muchas universidades públicas y privadas hicieron un replanteamiento de sus programas educativos para implementar en ellos el enfoque de la educación basada en competencias (Anguas, 2013).

Desde la elevación al rango de licenciatura de todas las carreras normalistas en 1984, estas escuelas han sido objeto de diversos programas que buscan su transformación, aunque aún no se ha conseguido. Igualmente, han venido enfrentado diversas críticas respecto a la calidad de la formación que brindan a su estudiantado y a la calidad de sus propios cuerpos académicos (Instituto Nacional para la Evaluación de la Educación [INEE], 2016). Aunado a lo anterior, se justifica hoy más que nunca la necesidad de replantear nuevas estrategias para diversificar la formación docente en los procesos de reforma curricular actuales.

El modelo educativo que permea a los diferentes subsistemas de educación en nuestro país plantea una visión particular de la formación de quien enseña (Díaz Barriga, 2005) y, por ende, de las estrategias para su desarrollo profesional. Partiendo de esta premisa, el profesorado es considerado como una pieza clave en la consolidación de la calidad educativa, pues se constituye como el eje que facilita y guía el aprendizaje del estudiantado. Por estas razones, el perfil docente ostenta tres saberes estratégicos en su formación: el cúmulo de conocimientos disciplinares y pedagógicos (teórico); las habilidades para aprender de forma autónoma (heurístico); y el arreglo de actitudes y valores que requieren para detonar el aprendizaje del estudiantado de acuerdo a sus contextos (axiológico). De esta forma, este modelo ubica al docente como un agente mediador (Saint-Onge, 2000), con la capacidad y la responsabilidad para construir los ambientes de aprendizajes necesarios para que, de forma operativa, implemente en su extensión el currículo. 


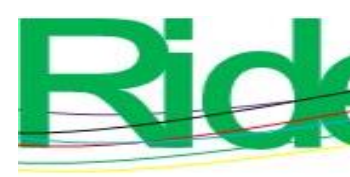
Revista Iberoamericana para la
Investigación y el Desarrollo Educativo
ISSN $2007-7467$

De aquí parte esta idea de que las escuelas normales transiten a estos esfuerzos inicialmente administrativos y de visión, a instituciones consistentes que consideren aspiraciones de perfil profesional con una visión periférica y transversal.

Vale la pena reconocer que existen cambios curriculares en los programas de estudio que vienen reformando el perfil profesional del maestro normalista, como es el caso de los planes y programas de estudio 2012 y 2017, donde se han revisado y modificado algunos programas académicos, considerando otros aspectos pedagógicos y didácticos. De hecho, se han discutido fuertemente a nivel nacional elementos sustantivos como el trayecto formativo del aprendizaje del inglés, el uso de las TIC y la falta de cursos sobre didácticas de las escuelas multigrado, educación inclusiva e intercultural, todo ello como un reflejo de las necesidades contextuales. Sin embargo, y de frente a las orientaciones que las universidades públicas estaban ya haciendo de forma paulatina y progresiva, aún queda pendiente transitar a la aspiración de trascender a contextos internacionales que movilicen estos aprendizajes en contextos internacionales.

Por otra parte, hay que destacar que la investigación juega un papel importante para la formación inicial del profesorado, pues promueve la mejora de la enseñanza y la reforma escolar, respondiendo a preguntas relacionadas con la observación y la reflexión, la experiencia y la experimentación (Rodríguez, 2004). Las recientes reformas educativas de 2013 en educación básica, aunadas a los constantes cambios en la política pública del sistema educativo nacional, han impactado significativamente en la estructura y dinámica académica de las escuelas normales en la última década. Desde hace varios años, la Dirección General de Educación Superior para Profesionales de la Educación (DGESPE) —órgano que dicta las políticas, planes y programas y en general la normatividad de las instituciones de educación superior (IES) dedicadas a la formación de profesionales en este ámbito — ha generado diversas iniciativas para profesionalizar la formación del magisterio a nivel nacional. A finales de la década de 1990, a través del programa para la transformación de las escuelas normales, se modificaron los planes de la licenciatura en educación primaria, preescolar y secundaria y se implementó la educación física y educación especial (Mercado, 2000).

Esta transformación — que se unía estratégicamente con el acuerdo nacional para la modernización de la educación básica generado desde 1992 — suponía la necesidad de aspirar a la construcción de un perfil académico normalista de profesor-investigador, por un lado, para satisfacer las demandas del gremio por revalorizar su profesión y, por el otro, para mejorar las 


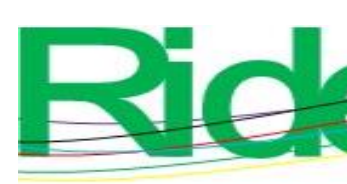

Revista Iberoamericana para la Investigación y el Desarrollo Educativo ISSN 2007 - 7467

condiciones laborales que desde hacía varios años lideraba el Sindicato Nacional de Trabajadores de la Educación (SNTE).

La nueva política educativa nacional que se perfilaba hacia principios del presente siglo comenzó a integrar a las escuelas normales al Programa de Mejoramiento del Profesorado (Promep), hoy Programa para el Desarrollo Profesional Docente (Prodep), de la Secretaría de Educación Pública (SEP), teniendo como célula básica de operación académica a los cuerpos académicos (CA), cuyo objetivo principal es equilibrar las funciones de docencia, investigación y gestión-vinculación, intentando dar a las escuelas normales un giro en los procesos identitarios y en la conformación de la disciplina de la profesión académica (Magaña, 2000).

La incursión de las escuelas normales al Prodep no se ciñe solamente a la promoción de esta diversificación de la carga docente-investigativa, sino a la posibilidad de acceder a programas de posgrado de excelencia a nivel nacional e internacional, la retención y repatriación de investigadores, la generación de recursos a través de proyectos y, de manera estratégica, la participación de la academia en proyectos de movilidad en el marco de los CA. Los resultados de la política educativa que integra el Prodep son importantes, pues implican la obtención de grados académicos, el incremento en el número de publicaciones, la obtención de recursos para la investigación (aunque la mayoría de las escuelas normales hayan quedado fuera de estos apoyos desde 2014) y aquellos rubros que están relacionados con la obtención de incentivos y permiten a la academia no solo mejorar su nivel salarial, sino también su prestigio (Romo, 2006).

Las transformaciones de fondo que se han realizado al sistema de las escuelas normales - y en particular la aspiración de mejorar el perfil del maestro normalista para adaptarse a una nueva necesidad de formación en concordancia con las tendencias nacionales e internacionalesobligan a las entidades gubernamentales, a las redes y los organismos formados a partir del gremio magisterial, a las propias escuelas normales y a la academia normalista a voltear la vista hacia procesos de formación, de cooperación y gestión relacionados con la globalización de la educación superior.

El conjunto de tendencias que empujan la transformación de las escuelas normales y específicamente al perfil del docente provienen en buena medida de enfoques nacionales, pero sin duda se complementan con aquellas que emiten los organismos internacionales a través de declaraciones, guías e informes de largo alcance. La OCDE, por ejemplo, aunque procura ser más incluyente en las recomendaciones hechas en materia de política educativa, no es ajena a la influencia de la globalización económica que impera en la actualidad. Los tres elementos 


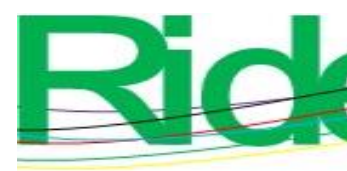

Revista Iberoamericana para la Investigación y el Desarrollo Educativo ISSN $2007-7467$

indispensables que establece para la educación son alfabetización, formación docente, así como enseñanza y formación técnico-profesional. En el nivel internacional, uno de los documentos más importantes que la Unesco ha publicado y que ha influido en la reflexión sobre la idea de que la educación debe seguir considerando las tendencias sociales, económicas, tecnológicas y políticas de este siglo es el Informe a la Unesco de la Comisión Internacional sobre la Educación para el Siglo XXI. Este documento es un análisis sobre la filosofía educativa como resultado de la situación política y económica que distingue a la globalización, pero también es una recomendación para adaptar los sistemas educativos a una dinámica de absorción cultural, tecnológica, económica, social, política (Fernández, 2005).

Tomando en cuenta los hitos recientes en los modelos estructurales de la escuela normal mexicana y la serie de retos que surgen a partir del crisol de influencias a nivel nacional e internacional, se debe comenzar a hablar del proceso de internacionalización en el modelo educativo de las escuelas normales mexicanas y, paralelamente, analizar cómo este elemento podrá contribuir a la consolidación de la calidad en la formación del profesorado, a la pertinencia global del perfil académico normalista, a la construcción de redes de cooperación con orientación a la investigación educativa y, por consiguiente, al fortalecimiento del desempeño académico y profesional de quien egresa de las normales en nuestro país.

Por ello, hablar de internacionalización en el campo de la educación es tener una variedad de elementos que hoy en día cobran mayor relevancia (González, 2007); es decir, hablar de los programas de estudio con pertinencia global, procesos de enseñanza-aprendizaje con enfoque intercultural, uso de lenguas extranjeras en la diversidad de actividades académicas, producción de investigación de forma conjunta con pares internacionales, generación de proyectos de gestión y/o vinculación a nivel internacional, presencia del estudiantado y profesorado visitantes, una variedad de acuerdos interinstitucionales y, por supuesto, la incursión de sus estudiantes y académicos/as en programas de movilidad.

Desafortunadamente el sistema educativo nacional, de forma generalizada, aún tiene un largo camino por recorrer en materia de agenda y especialmente de financiamiento para los procesos de internacionalización. A pesar de los esfuerzos que ha realizado la SEP a través de la Subsecretaría de Educación Superior, la Secretaría de Relaciones Exteriores con su diversificada oferta de programas y proyectos de cooperación internacional, la Asociación Nacional de Universidades e Instituciones de Educación Superior (ANUIES) y los diferentes consorcios y asociaciones independientes que han desarrollado estrategias, documentos y declarativas en 


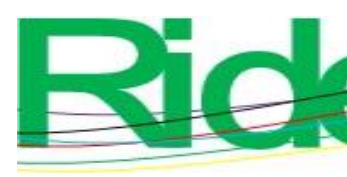

Revista Iberoamericana para la Investigación y el Desarrollo Educativo ISSN $2007-7467$

donde ubican a la internacionalización como una estrategia insoslayable para los nuevos retos de la educación en México, aún no existe una política definida y de largo plazo que logre orientar a las instituciones de educación superior sobre la serie de acciones y mecanismos sobre los cuales deberán de transitar para consolidar la dimensión internacional en sus modelos y estructuras.

De igual forma, al igual que en otros aspectos igualmente importantes en el ámbito educativo como la investigación, la vinculación y la infraestructura, el financiamiento para las acciones de internacionalización en las instituciones educativas mexicanas depende, en buena medida, de coyunturas financieras que emiten de forma intermitente las administraciones federales y de los esfuerzos que las propias administraciones de las instituciones educativas realizan de acuerdo con sus capacidades y agendas directivas y rectorales.

A pesar de la inexistencia de una política nacional clara sobre incorporación de la dimensión internacional en la agenda educativa en México, las instituciones en lo individual han caminado a diferentes ritmos. Algunas universidades privadas, por ejemplo, han incorporado desde hace décadas planes y estrategias que las han colocado hoy por hoy como líderes en indicadores de internacionalización: número de estudiantes en movilidad, programas de doble titulación, campus transfronterizo, reclutamiento de estudiantes internacionales, currículos en otros idiomas, entre otros. En menor medida, pero con la fortaleza de presupuestos robustos y constantes, están las grandes universidades públicas mexicanas, que gracias a su presencia, tradición de docencia e investigación y a la posibilidad de poseer estructuras de gestión que posibiliten el proceso de internacionalización, han tenido logros importantes en muchos de los indicadores de gestión y desempeño en tal rubro. De acuerdo con Gacel-Ávila (2005), una dimensión internacional proviene de un conjunto de condiciones, tales como el grado de internacionalización de su currículo, el perfil internacional de sus académicos, la competencia global de quienes egresan y, de manera general, un ethos internacional en la institución.

En cambio, la mayoría de instituciones educativas que no están en los segmentos privilegiados anteriormente mencionados han tenido avances muy limitados en materia de internacionalización, proceso al que han llegado tarde y sin un plan estructurado de acción, por lo que eminentemente han venido implementando acciones inerciales y con base en la experiencia de otras instituciones. Al igual que otros procesos de trasformación y fortalecimiento, para el caso de las escuelas normales se incorpora la internacionalización de manera tardía (2009), en un ejercicio de imposición de ideas, modelos y concepciones universalizadas (Noriega-Chávez, 2014). 


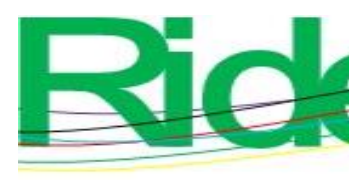

Revista Iberoamericana para la

Investigación y el Desarrollo Educativo

ISSN $2007-7467$

\section{La movilidad académica en la formación integral}

Como ya lo hemos apuntado, es la globalización de la economía, la movilización de la información y la apertura de las barreras comerciales los principales factores que desencadenaron el surgimiento de la sociedad del conocimiento. Como apunta Martín-Barbero (2003), este conocimiento circula en dichas sociedades con mayor libertad, puesto que se maneja como información, y la producción y legitimación de saberes no se limita a un círculo social determinado rígidamente, situación que deriva en la transformación de la sociedad y del espacio escolar en todos sus niveles.

El incremento de la cooperación internacional y de los flujos estudiantiles a nivel mundial coincide con el advenimiento de una mayor movilidad de información y conocimientos que se apoya y en muchos casos se sustenta en la utilización de las tecnologías de la información y la comunicación, las cuales han hecho posible la formación de canales de comunicación y contacto con variadas expresiones culturales, formas de vida y de trabajo académico que permitieron la creación de escenarios de trabajo, investigación y desarrollo conjunto alternativos e innovadores (García, 2013).

Para hablar de movilidad académica, debemos de analizar el marco conceptual del cual se desprende. Mucho se habla actualmente sobre programas de movilidad y de las bondades que esta produce en el desempeño académico tanto del estudiantado como del profesorado, pero muy pocas veces se le concibe como parte de un macroproceso llamado internacionalización de la educación superior. La internacionalización es comúnmente confundida con la globalización (Altbach, 2004). Los resultados de la globalización en el ámbito de la educación incluyen la integración de la investigación aplicada, el uso del inglés como lengua franca en la comunicación científica, el crecimiento del mercado laboral para académicos y científicos, el crecimiento de empresas y multinacionales que ofrecen tecnología y servicios para publicar productos académicos y el uso de las tecnologías de la información (Altbach y Knight, 2007). En cambio, la internacionalización - aunque obedece a diversos factores y necesidades que resultan de aspectos políticos, económicos, socioculturales y académicos (De Witt, 2002)—ofrece un acercamiento más apegado a las adaptaciones de fondo que deben tener las funciones sustantivas de toda institución educativa ante la inexorable tendencia de la globalización del conocimiento. De esta forma, podemos decir que, en apego a la orientación académica de la internacionalización, esta se puede definir como el desarrollo de las dimensiones internacionales 


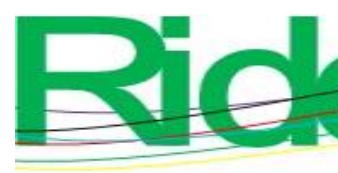

Revista Iberoamericana para la Investigación y el Desarrollo Educativo ISSN 2007 - 7467

e interculturales en la investigación, en la docencia y en los servicios para el mejoramiento de la calidad y de los estándares académicos internacionales (Zaman y Mohsin, 2014).

La movilidad académica implica en términos generales el desplazamiento físico de una persona para realizar actividades académicas en una institución de educación superior distinta, lo cual puede suceder en un contexto regional, nacional e internacional. En muchas ocasiones, aun cuando se hace constantemente hincapié sobre las diferencias entre la movilidad y el proceso de internacionalización, la primera sigue siendo vista como la base o la versión operativa de la segunda, lo cual es una concepción errónea y muy limitada.

Para concebir a la movilidad es preciso destacar primero las implicaciones de una estancia académica. En palabras de Ruiz (2011), es una estrategia educativa de carácter curricular que permite al estudiante un proceso de formación para incorporarse profesionalmente a los diversos sectores productivos de bienes y servicios, a través del desarrollo de un proyecto definido de trabajo profesional, con lo que se busca que el estudiantado se vea enfrentado a situaciones que posiblemente rebasen su nivel de conocimientos; de esa forma, ellos se verán obligados a desarrollar su capacidad analítica y de impulso para investigar por su cuenta, demandar mayores explicaciones e interactuar con el profesorado. En otras palabras, es llevar a estudiantes mediante la estancia académica a un proceso in situ de complejidad analítica y de resolución de problemas en contextos diversos y multifactoriales como una forma estratégica de promover la formación integral.

Aspirar a este tipo de formación, según Díaz y Quiroz (2013) implica la preparación social e individual de las personas, tanto científica como ideológica y espiritualmente, válida para una época histórica donde todo proceso de formación procura en el estudiantado desde las diferentes disciplinas ligar los contenidos de la enseñanza con su significación cultural, ética y estética, buscando fortalecer la capacidad humana de la comunicación.

Las estancias de movilidad académica propiamente tienen finalidades más específicas y con mayor profundidad formativa, entre las que destacan ayudar a estudiantes - a través de una experiencia académica con gran diversidad intercultural, interétnica y social — para que puedan fomentar el pensamiento independiente, la capacidad de adaptación a la diversidad cultural, a eliminar barreras de comunicación, a expandir la visión profesional y, entre muchos otras cosas, a generar las bases de la ciudadanía global. 


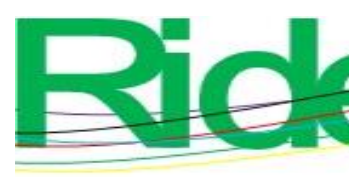

Revista Iberoamericana para la Investigación y el Desarrollo Educativo ISSN 2007 - 7467

En pocas palabras, la movilidad académica hace referencia al desplazamiento de investigadores, docentes y estudiantes entre instituciones educativas nacionales y extranjeras con el objetivo de participar en programas formativos y proyectos de investigación particulares. La movilidad académica suele estar situada dentro de un esquema de trabajo interinstitucional, nacional o internacional, aunque es posible que el flujo de personas se desarrolle a partir de la iniciativa de los propios sujetos o como parte de proyectos específicos que no necesariamente involucran el trabajo con varias instituciones o centros de investigación (García, 2013).

Parafraseando a Fresán (2009), movilidad académica es una estrategia formativa cuyo objetivo rebasa el ámbito estrictamente académico y se refiere principalmente a la formación integral. Es deseable que la experiencia sea enriquecedora en muy diversos ámbitos, porque además de los desafíos que representa su incorporación a un entorno universitario diferente, con una cultura distinta y otros modelos de enseñanza-aprendizaje, se agregan los de hacerse cargo de sí mismo como individuo, tanto en lo que se refiere a su cuidado personal como a la cautela en el uso de recursos económicos y a la necesidad de convivir con el resto de la gente fuera del ámbito universitario. Por ello, una acción sustantiva que no debemos pasar inadvertida en la formación del estudiantado es, además de la formación académica, su independencia personal y socioemocional, que en estos tiempos cada vez más nos lo demandan.

Muchas instituciones de educación superior del mundo han implementado programas de intercambio y movilidad estudiantil con la finalidad de ayudar a jóvenes universitarios/as a desarrollar competencias que mejoren su perfil profesional y les ayuden a interactuar más eficazmente en el ambiente globalizado en que vivimos. Estos programas están basados en la posibilidad de que quien estudia se integre temporalmente a una institución diferente a la suya, cursando asignaturas para la transferencia de créditos académicos para realizar actividades de investigación o para realizar programas especiales de verano para aprender lenguas o ciertas competencias a nivel disciplinar. La movilidad académica constituye, entonces, una de las herramientas que muchas universidades e instituciones alrededor del mundo utilizan de forma estratégica para elevar la calidad educativa, ya que permite que el estudiantado desarrolle competencias diferenciadas que den respuesta a las demandas sociales del entorno local, regional y también global.

La movilidad de académicos/as y del estudiantado de las escuelas normales representa una alternativa para elevar la calidad académica y la competitividad entre toda su comunidad educativa (estudiantes, docentes e investigadores/as), propiciando la estancia en otras 


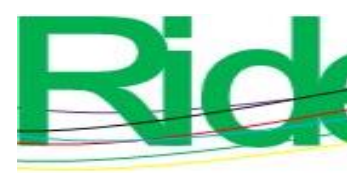

Revista Iberoamericana para la Investigación y el Desarrollo Educativo ISSN 2007 - 7467

instituciones de educación superior de México o del extranjero con el objeto de cursar semestres completos, realizar prácticas profesionales, participar en proyectos de investigación y/o recibir asesoría para la conclusión de sus trabajos de titulación. Estos programas favorecen la formación profesional, aumentan la cohesión académica y estudiantil del país, contribuyen a elevar el nivel de excelencia académica y mejoran la comunicación entre quienes investigan, además de incentivar la participación del estudiantado en proyectos multidisciplinarios.

Aunado a los beneficios formativos que representa la inclusión de la movilidad académica en las escuelas normales, es necesario resaltar que esta estrategia de internacionalización tenga un impacto directo en la construcción de un perfil intercultural del futuro profesional de la educación. De acuerdo con Deardorff y Hunter (2006), el centro de los enunciados de misión de las instituciones de educación superior actualmente tienen como fundamento el preparar graduados "globalmente listos", los cuales deben ser capaces de lidiar con retos de orden global. Sin embargo, nuestras ineficientes instituciones poco están haciendo sobre este respecto (Bok, 2006). Esto trae a la competencia intercultural y a la diversidad al escenario principal de la necesidad de abordar la internacionalización y la movilidad como ejes fundamentales de la adquisición de modelos educativos con enfoque global.

Por estas y otras razones de orden académico-social, se asume que no hay duda de que la internacionalización y particularmente la movilidad de estudiantes han transformado el panorama de la educación superior en las últimas décadas. Esta movilización de personas con objetivos académicos ha traído diversos beneficios a estudiantes, instituciones, comunidades y a los países en su conjunto (Knight, 2012).

\section{Indicadores sobre movilidad estudiantil}

El número de estudiantes extranjeros que participan en programas de educación superior de todo el mundo se ha disparado en una sola generación, al incrementarse desde 0.8 millones a finales de los años setenta a 4.6 millones (es decir, 45 años más tarde). Tan solo en 2015 hubo 3.3 millones de estudiantes que se desplazaron por el mundo con fines educativos (OCDE, 2018).

Los grupos y flujos de estudiantes móviles siguen estando muy concentrados; además, las vías de movilidad están profundamente arraigadas en modelos históricos y determinados por factores de proximidad. Los cinco principales países de destino de la OCDE reciben a casi $70 \%$ del estudiantado en movilidad dentro de la zona de la OCDE, mientras que los cinco primeros países de origen (en todo el mundo) representan poco menos de 40 \% de la migración total hacia 


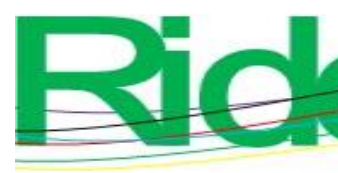

Revista Iberoamericana para la Investigación y el Desarrollo Educativo ISSN 2007-7467

esa zona. Los países que mayormente son receptores tienen economías avanzadas y son de habla inglesa: Estados Unidos (30\% del total de estudiantes internacionales en la zona de la OCDE), Reino Unido (14\%) y Australia (10\%). No obstante, Alemania, Federación Rusa y Francia también atraen a un número considerable de estudiantes. La mayoría del estudiantado en movilidad dentro de los países de la OCDE proceden de China (20\%), seguidos de India (7 \%), Alemania (4\%), Corea, Francia y Arabia Saudita (que se sitúan en 2 \% y 3 \%) (OCDE, 2018).

En América Latina y el Caribe (ALC) la movilidad (principalmente a nivel licenciatura y posgrado que realizan estudios completos) es una actividad que las IES han apoyado en cierta medida. Los flujos más numerosos de estudiantes latinoamericanos/as salientes se dirigen a espacios educativos ubicados en Estados Unidos y después en España, Alemania, Francia y Gran Bretaña. Una tendencia sobresaliente en la década en curso es el retour en force de Estados Unidos como principal imán para estudiantes de la región. Su número pasó de 65632 en 20092010 a 86378 en 2014-2015 (aumentó en 31.6\% en el periodo) (Didou, 2017). La tasa de participación de ALC en el total de movilidad entrante a Estados Unidos fue de 8.9 \% en 20142015. Brasil (en sexta posición) y México (en décimo rango) están entre los diez países que más envían estudiantes a ese país (Didou, 2017), seguidos por Venezuela y Colombia.

En México hay 3.8 millones de estudiantes inscritos en cerca de 3500 IES, de las cuales solo $10 \%$ reportó en la base 911 haber tenido al menos un estudiante participando en un programa de movilidad en el extranjero durante el ciclo escolar 2015/2016 (SEP, 2017). Los datos son aún más desalentadores al observar el número total de estudiantes de movilidad, que dependiendo de la fuente consultada - la encuesta PATLANI o la base 911 de la SEP — fueron entre 23000 y 30 000. En México apenas entre $0.6 \%$ y $0.8 \%$ de los estudiantes de nivel superior tienen la oportunidad de realizar actividades temporales de índole académica en el extranjero.

La Encuesta Nacional de Movilidad Internacional Estudiantil PATLANI se ha consolidado como una fuente de información valiosa y confiable para organizaciones nacionales e internacionales, investigadores, catedráticos e instituciones de educación superior interesados en el desarrollo de la movilidad estudiantil como parte fundamental de la internacionalización, eje trascendental del desarrollo de la educación superior en nuestro país. La tabla 1 presenta los flujos de movilidad que ha tenido nuestro país en el periodo entre 2011 y 2016. 
Tabla 1. Resumen de la Encuesta Nacional de Movilidad Internacional Estudiantil, PATLANI

\begin{tabular}{|l|l|l|l|l|l|}
\hline $\begin{array}{l}\text { CICLO } \\
\text { ESCOLAR }\end{array}$ & $\mathbf{2 0 1 1 / 2 0 1 2}$ & $\mathbf{2 0 1 2 / 2 0 1 3}$ & $\mathbf{2 0 1 3 / 2 0 1 4}$ & $\mathbf{2 0 1 4 / 2 0 1 5}$ & $\mathbf{2 0 1 5 / 2 0 1 6}$ \\
\hline $\begin{array}{l}\text { MOVILIDAD } \\
\text { SALIENTE }\end{array}$ & 17,689 & 15,941 & 20,599 & 24,900 & 29,401 \\
\hline $\begin{array}{l}\text { MOVILIDAD } \\
\text { ENTRANTE }\end{array}$ & 8,795 & 18,125 & 16,685 & 15,608 & 20,116 \\
\hline
\end{tabular}

Fuente: Castiello, Cortes y Bustos (2018)

Los resultados de movilidad estudiantil internacional en México obtenidos en PATLANI (en los formatos 911, principalmente) y en otras fuentes de información adicionales en relación con dos periodos (2014/2015 y 2015/2016) son los siguientes:

Número de estudiantes de movilidad: En el periodo 2014-2015 se registraron 24900 estudiantes en movilidad saliente y 15608 de movilidad entrante. Para el lapso 2015-2016 se reportaron 29401 estudiantes en movilidad saliente y 20322 en movilidad entrante. En cambio, los formatos 911 reportan en 2014-2015, 16182 estudiantes de movilidad saliente y 7201 de entrante (2014-2015), cifra que aumenta a 22988 en movilidad saliente y a 8492 la movilidad entrante en el ciclo siguiente (2015-2016). De estos datos se desprende que la movilidad estudiantil ha crecido a lo largo de las ediciones de PATLANI y de los registros de los formatos 911 (a pesar de sus disparidades en cifras).

Proporciones de movilidad en relación con la matrícula general: Tomando como referencia la matrícula general de las IES que reportaron a PATLANI, en promedio 1\% corresponde a movilidad saliente en cualquiera de los dos periodos. Lo mismo ocurre con la movilidad entrante. En esa misma proporción, pero tomando como fuente de información los formatos 911, los resultados son más pesimistas: la movilidad entrante corresponde a $0.2 \%$ en cualquiera de los dos periodos, mientras que la saliente es de $0.4 \%$ en el $2014-2015$ y $0.5 \%$ en el 2015-2016.

Movilidad por tipo de institución (públicas o privadas): En el ciclo 2014-2015, la mayor parte de la movilidad entrante y saliente se dio en IES privadas. Para el 2015-2016, la mayor parte de la movilidad se da en IES públicas. Esta tendencia se observa tanto en PATLANI como en la información de los formatos 911.

Principales IES mexicanas: En movilidad saliente, el Instituto Tecnológico y de Estudios Superiores de Monterrey (ITESM) fue la institución que presentó mayor movilidad en ambos periodos. En relación con la movilidad entrante, en el 2014-2015, la principal institución fue 


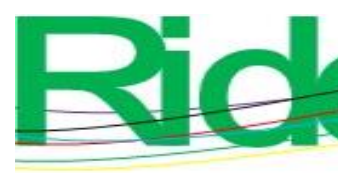

Revista Iberoamericana para la Investigación y el Desarrollo Educativo ISSN $2007-7467$

nuevamente el ITESM; sin embargo, para el 2015-2016 se reportó mayor movilidad en la Universidad Nacional Autónoma de México (UNAM). Un tema que se evidencia en este reporte es la poca comparabilidad que existe entre las primeras veinte IES que reportan movilidad en PATLANI y las incluidas en la base 911. Hay cierta coincidencia en la importante movilidad que existe en el ITESM, pero existe una fuerte disparidad con respecto al resto de las instituciones entre las dos fuentes de información.

Principales campos de formación: Como ha sucedido en todas las ediciones de PATLANI, el campo de formación más importante en ambos periodos reportados y para movilidad entrante y saliente es el de ciencias sociales, administración y derecho.

Movilidad estudiantil en América del Norte: En esta región se registran 6832 estudiantes de movilidad saliente en el 2014-2015, cifra que disminuyó a 6701 estudiantes en 2015-2016. Con ello, se constata como la segunda región más importante después de Europa. En la movilidad entrante periodo 2014-2015, 3681 estudiantes fueron reportados, cifra que aumentó a 4510 estudiantes en el 2015-2016. En el análisis, se detectan relaciones desiguales entre la movilidad estudiantil de México con los países de esta región: México envía más estudiantes de los que recibe, mientras que el tipo de movilidad que realizan es diferente entre estos países. La relación indica que por cada 6 estudiantes mexicanos que van a Canadá, un estudiante canadiense viaja a México; mientras que por cada estudiante estadounidense que viene a México, 1.4 estudiantes viaja a Estados Unidos.

Movilidad estudiantil en Europa: Se trata de la región más importante en cuanto a movilidad estudiantil para México. En el 2014-2015 salieron 12901 estudiantes mexicanos, y esta cifra aumentó a 17763 en el 2015-2016. En la movilidad entrante (2014-2015) se reportaron 5099 estudiantes a IES mexicanas, lo que en el siguiente periodo ascendió a 6280. En esta región también se observa movilidad estudiantil asimétrica en su mayoría, sobre todo con España (por cada 6 mexicanos que van a España uno viene), Reino Unido (por cada dos alumnos que van a Reino Unido uno viene a México) e Italia (por cada 4 alumnos mexicanos que van a Italia un italiano viene a México). En el caso de Alemania y Francia, la relación es un poco más pareja. Lo anterior plantea retos a las IES mexicanas por instrumentar estrategias de largo plazo que permitan movilidad estudiantil más bilateral.

Movilidad estudiantil en América Latina y el Caribe: De entre las tres regiones analizadas, se trata de la de menor afluencia en movilidad estudiantil. Para 2014-2015, la movilidad saliente fue de 4278 estudiantes, lo que aumenta a 5911 en 2015-2016. En la movilidad entrante 2014- 


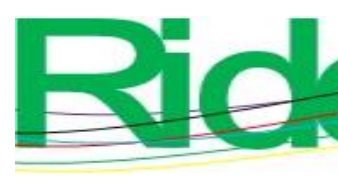

Revista Iberoamericana para la Investigación y el Desarrollo Educativo ISSN $2007-7467$

2015, se registraron 5456 estudiantes, dato que incrementa a 7063 en 2015-2016. Esta región constituye una zona de mayor equilibrio en cuanto al envío y la recepción de estudiantes con IES mexicanas. No obstante, la principal afluencia de movilidad se realiza en el marco de determinados países. Por ejemplo: por cada 2 colombianos que vienen a México, un mexicano va para allá; por cada dos mexicanos que van a Argentina, un argentino viene a México, y por cada cinco mexicanos que van a Chile, un chileno viene a México, principalmente.

\section{Escenarios de movilidad para las escuelas normales}

Es innegable el avance que la internacionalización y la movilidad estudiantil ha tenido a lo largo de las últimas décadas en la vida de las instituciones de educación superior, incluso en la región de Latinoamérica, en donde sabemos de la existencia de un rezago importante en términos de acceso, infraestructura, financiamiento y política pública en materia educativa.

Tal como lo vemos en las estadísticas descritas en el apartado anterior, México muestra ciertos avances en la participación de estudiantes en movilidad, sin embargo, es claro que el trecho por recorrer es amplio, sobre todo cuando el ámbito de la internacionalización en su conjunto está aún por consolidarse en nuestro país.

Las escuelas normales no han escapado a esta tendencia de mover estudiantes y abrirse hacia esquemas de cooperación que coadyuven al incremento de su calidad educativa. Si bien es reducido el número de escuelas normales que participan sistemáticamente en programas de movilidad, la demanda e interés por este tipo de herramientas de formación continúa creciendo. Instituciones normalistas de los estados de Puebla, Jalisco, Durango, Estado de México, Veracruz, Baja California Sur, Michoacán y Tlaxcala, entre otros, han logrado que un número acotado de estudiantes y académicos accedan a alguna oportunidad para realizar una estancia de movilidad dentro o fuera de México, aunque actualmente no se puede conocer a ciencia cierta el flujo exacto de la movilidad saliente y entrante por institución o por estado, así como los principales destinos y los mecanismos por los cuales cada estudiante está realizando dichas estancias.

En la actualidad, podemos dar cuenta de varios programas y convocatorias eminentemente de fondos federales que promueven la movilidad de estudiantes y profesores de escuelas normales, por lo que podemos destacar los siguientes: 


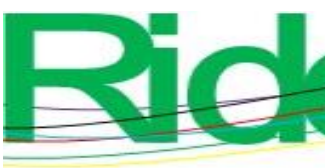

Revista Iberoamericana para la Investigación y el Desarrollo Educativo ISSN 2007 - 7467

- Programa Interjom (intercambio para jóvenes maestros).

- Programa de capacitación académica para estudiantes y docentes miembros del cuerpo directivo de escuelas normales en Francia (SEP-CRFDIES).

- Beca en el Programa de Capacitación para Estudiantes y profesorado de Escuelas Normales Públicas (DGESPE-CNBES)

- Movilidad Académica Internacional Proyecto Paulo Freire en Países de Iberoamericanos dirigida a estudiantes de Escuelas Normales Públicas de México.

- Programa de Capacitación de Estudiantes SEP-SRE Proyecta 100000.

- Beca Programa para el desarrollo de competencias lingüísticas y comunicativas del inglés, promovida por la SEP, y DGESPE.

- Convocatoria de becas Elisa Acuña, Movilidad Estudiantil Nacional para escuelas normales.

- Programa de Movilidad Nacional Escuelas Normales y Universidades Públicas SEP.

- Programa Integral de Fortalecimiento Institucional (PIFI).

- Programa para el desarrollo profesional docente (Prodep).

Estos programas, junto con la mayoría de las iniciativas que gobiernos y organismos ofrecen al estudiantado mexicano y particularmente a aquel de las escuelas normales, están basados en la modalidad de estancia de movilidad temporal por créditos, la cual es la forma más común de concebir y promover la movilidad en el país y en nuestra región. Sin embargo, actualmente existen diversas formas de operar y organizar la movilidad de estudiantes. De manera contraria, en países como Estados Unidos o aquellos del continente europeo, cuando se habla de movilidad estudiantil normalmente se refieren a aquellos estudiantes que están realizando estudios completos de educación superior en un país diferente al de origen, y en menor medida a estudiantes que realizan estancias semestrales, comúnmente llamados Study Abroad programs.

Lo cierto es que la movilidad no solo implica el realizar una estancia que involucre créditos académicos, independientemente de su duración. La movilidad poco a poco se ha diversificado en acciones que implican las prácticas de campo, estancias de laboratorio, programas de doble titulación y recientemente actividades de movilidad virtual. Existen seis categorías para organizar la movilidad estudiantil que obligan ir más allá de los conceptos de estancia semestral o estudios completos en el extranjero (Knight, 2012): 


\begin{tabular}{|c|c|c|c|}
\hline $\begin{array}{l}\text { Investigación } \\
\text { trabajo } \\
\text { campo }\end{array}$ & $\begin{array}{r}\text { y } \\
\text { de }\end{array}$ & $\begin{array}{l}\text { Estancia de investigación o trabajo de } \\
\text { campo como requisito para la titulación en } \\
\text { la institución de origen. }\end{array}$ & $\begin{array}{l}\text { La institución de origen } \\
\text { emite el título } \\
\text { cumpliendo el requisito } \\
\text { de la estancia. }\end{array}$ \\
\hline $\begin{array}{l}\text { Internados } \\
\text { prácticas } \\
\text { profesionales }\end{array}$ & $\mathbf{y}$ & $\begin{array}{l}\text { Estancia como practicante, internado o } \\
\text { servicio comunitario como requisito para } \\
\text { obtener el título en la institución de origen. }\end{array}$ & $\begin{array}{l}\text { La institución de origen } \\
\text { emite el título } \\
\text { cumpliendo el requisito } \\
\text { de la estancia. }\end{array}$ \\
\hline $\begin{array}{l}\text { Viajes } \\
\text { estudios } \\
\text { asistencia } \\
\text { talleres. }\end{array}$ & de & $\begin{array}{l}\text { Estancias opcionales y en ocasiones } \\
\text { requisitos dentro del plan de estudios. } \\
\text { Pueden ser estancias de verano, programas } \\
\text { culturales o cursos de lengua. }\end{array}$ & $\begin{array}{l}\text { Normalmente no generan } \\
\text { créditos académicos. }\end{array}$ \\
\hline
\end{tabular}

Fuente: Six categories of student mobility experiences (Knight, 2012)

De esta forma, podemos advertir que otro de los retos que enfrenta nuestro sistema educativo mexicano es justamente diversificar las formas y las modalidades en las que gestionamos y promovemos la movilidad académica.

Existen ya experiencias exitosas en diversas universidades públicas y privadas en México, en donde se practican desde hace algunos años programas de movilidad menos tradicionales, como lo son los programas twining (Programa de hermanamiento académico, donde el estudiantado puede transferir sus créditos de una institución a otra y tener la posibilidad de graduarse en una o ambas instituciones), los programas de doble titulación o los programas de franquicia. La Universidad Nacional Autónoma de México, con sedes y programas filiales en Estados Unidos, España, Alemania, Francia, China, Canadá, Sudáfrica y Reino Unido, o el ITESM, con cerca de 14 oficinas representativas en distintos lugares del mundo, además de los numerosos programas en donde ofrecen programas de doble titulación impartidos en lengua extranjera y con alta presencia de estudiantes internacionales son sin duda las universidades líderes en el establecimiento de programas franquicia y educación transfronteriza en nuestro país. De igual forma, se encuentran otras universidades mexicanas que le han apostado a la diversificación de las modalidades de la movilidad académica, impulsando programas conjuntos, dobles titulaciones, programas filiales con universidades extranjeras, carreras o programas educativos tipo franquicia en México, además de una inmensa oferta de programas de duración 


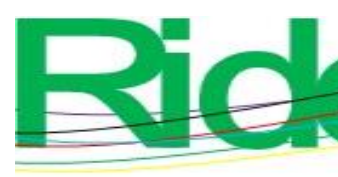

Revista Iberoamericana para la

Investigación y el Desarrollo Educativo

ISSN $2007-7467$

corta, como lo son los veranos académicos, las estancias de investigación, las prácticas profesionales internacionales o los cursos de lengua. Las instituciones que podemos distinguir en este ámbito de manera enunciativa, mas no limitativa, son la Universidad de Guadalajara, la Universidad de Monterrey, la Universidad Autónoma de Ciudad Juárez, la Universidad de Guanajuato, la Universidad Veracruzana, la Universidad del Valle de México, la Universidad Anáhuac, la Universidad Autónoma Metropolitana, la Universidad Autónoma de Nuevo León, entre muchas otras instituciones de educación superior.

Las escuelas normales mexicanas tienen, con base en la experiencia de otras instituciones de educación en nuestro país, una excelente oportunidad para incursionar no solo en el ámbito de la movilidad académica de forma integral, sino también para comenzar a sentar las bases del proceso de internacionalización en su conjunto y permear positivamente a sus modelos educativos, a sus estructuras y por ende a sus estudiantes y académicos en las próximas décadas. Para lograr tal fin, las escuelas normales requieren replantear sus planes institucionales de largo plazo integrando perspectivas de orden académico (curricular), estructural (gestión) y financiero (fondos).

Pero antes de establecer rumbos de acción y realizar propuestas para apoyar la incorporación de las escuelas normales al espectro de la internacionalización y particularmente de la movilidad estudiantil surgen una serie de interrogantes sobre los elementos que guían y detonan la necesidad de internacionalizar una institución, así como las motivaciones para integrar la movilidad en la educación superior (De Witt, 2002). Describamos algunos escenarios que servirán para generar propuestas posteriormente:

Detonantes para incorporar la movilidad en las funciones sustantivas de las escuelas normales. Ya hemos analizado en buena medida la serie de impactos que la movilidad trae a los procesos de formación integral, la forma en la que esta contribuye a la construcción de un perfil intercultural, diferenciado y con valor agregado a estudiantes y la academia, así como la contribución que tiene al desarrollo de habilidades de orden personal en quienes deseen desplazarse a otros contextos sociales, académicos y culturales. Pero ¿qué lleva a la institución a decidir transformar su ethos e invertir recursos diversos en la movilidad?

En un estudio realizado a tres universidades españolas, Grasset (2013) concluye que existen seis motivaciones principales que orientan la búsqueda de una perspectiva más internacional y particularmente promover esfuerzos de movilidad académica: 1) el desarrollo de competencias profesionales de estudiantes y académicos/as; 2) generar alianzas estratégicas 


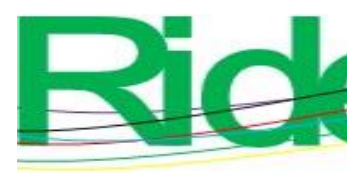

Revista Iberoamericana para la Investigación y el Desarrollo Educativo ISSN $2007-7467$

(redes); 3) obtener reconocimiento internacional: international branding (se refiere a la generación del concepto de "marca" institucional, como una forma de promover la venta de servicios educativos a nivel internacional); 4) incrementar la calidad educativa; 5) generar ingresos a través de programas internacionales y 6) desarrollar la responsabilidad social en estudiantes.

Las escuelas normales deberán de realizar un análisis profundo sobre las razones por las cuales desean y/o deben implementar acciones de movilidad académica, ya que dicho insumo será clave para el establecimiento de un alcance estratégico realista que determine metas y acciones específicas, la definición de programas, etapas de preparación y esquemas de financiamiento sostenido.

Alcances y beneficiarios de la movilidad en las escuelas normales. En este trabajo nos hemos referido de forma concreta al término movilidad académica, aunque la connotación común en nuestro país y en muchos otros de América Latina se refiere a la movilidad que hacen estudiantes y no necesariamente los académicos/as o las personas encargadas de funciones de gestión institucional. Será necesario identificar si las propuestas de acción programáticas para la movilidad serán solo para estudiantes, para académicos o para ambos, a la vez de establecer poblaciones objetivo con metas muy realistas y puntuales que nos permitan medir resultados y obtenerlos de forma gradual.

Sobre este punto valdría la pena hacernos la siguiente interrogante: ¿por qué insistimos en que la movilidad de estudiantes es prioritaria y no fomentamos de forma institucional la movilidad del profesorado?

Es bien sabido que la academia o docentes han tenido siempre la disposición y la voluntad de realizar estancias dentro y fuera del país. Son muchos los ejemplos de quienes han realizado diversas acciones de cooperación internacional, ya sea porque han cursado estudios de posgrado, participan en redes o proyectos internacionales, participan en eventos académicos, realizan cursos especializados o desarrollan algún protocolo de investigación en conjunto con algún par extranjero. Sin embargo, estas acciones son principalmente fruto del esfuerzo individual, o de algún cuerpo académico, más que por una política o programa de las instituciones de adscripción. La movilidad de académicos y científicos es autoorganizada, motivada por la necesidad de incrementar el prestigio y la credibilidad, además de ser producto del proceso de extender el espacio social individual, es decir, es estimulada por el deseo de la socialización profesional (Mahroum, 2000). 


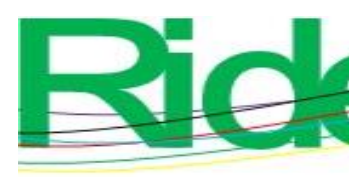

Revista Iberoamericana para la Investigación y el Desarrollo Educativo ISSN 2007 - 7467

Bajo esta perspectiva, será prudente promover en las escuelas normales mexicanas el equilibrio de esfuerzos institucionales para motivar tanto a estudiantes como a la academia no tan solo a participar en las opciones que tienen para realizar movilidad, sino a procurar los perfiles, los recursos económicos, los mecanismos administrativos y la planeación curricular para que la movilidad sea factible y deseable de forma transversal. El impulso de la movilidad de estudiantes será más complejo si la planta académica no tiene experiencia internacional, y para ello es necesario comenzar a crear sensibilidad y conciencia sobre la importancia que representa el realizar estancias académicas fuera de la institución, a nivel individual, pero sobre todo a nivel institucional.

\section{Planeación y habilitación institucional para la movilidad en las escuelas normales}

Una vez que hemos reiterado la importancia, necesidad y pertinencia de la implementación de los procesos de movilidad académica (de estudiantado y profesorado) en la vida institucional de las escuelas normales, hablaremos de un aspecto que ha sido poco abordado en la literatura relacionada a la internacionalización y que tiene gran relevancia en el éxito o fracaso de los esfuerzos por institucionalizar la movilidad, es decir, abordaremos las implicaciones de la gestión de la movilidad académica.

Desarrollar una política y un plan sostenido sobre internacionalización y específicamente sobre movilidad académica no es un asunto exclusivamente de voluntades, sino de procesos largos y consistentes de habilitación institucional que incluye factores administrativos, académicos, financieros, culturales e incluso políticos, ya que como lo hemos dicho la historia de las escuelas normales ha estado marcada por los constantes cambios de orden político que han movido al sistema educativo nacional en las últimas décadas.

Con la finalidad de brindar una base sobre la gestión estratégica que podrán implementar las escuelas normales y puedan identificar sus oportunidades en el rubro de movilidad académica y canalizar sus esfuerzos de forma integral, se presentan cuatro factores clave que se deberán desarrollar de forma paralela: estructura, planeación, currículo y financiamiento.

Estructura de gestión para la movilidad y la internacionalización. La operación y el seguimiento de los procesos de movilidad en una institución, si bien se pueden realizar desde lo individual (como es el caso de muchos estudiantes y académicos actualmente), se requiere de una figura (entidad) institucional que respalde académica, administrativa y financieramente a los 


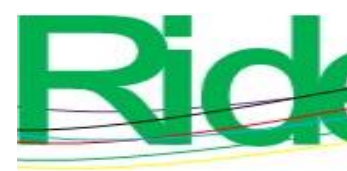

Revista Iberoamericana para la Investigación y el Desarrollo Educativo ISSN 2007 - 7467

universidad. Para alcanzar dicho objetivo se procura tener una visión más general del proceso de gestión, que comprenda las diversas dimensiones de este proceso atravesadas por las siguientes variables seleccionadas: planificación estratégica, gestión de programas y proyectos, procesos de evaluación y monitoreo, decisión política de las autoridades universitarias, la capacitación del personal que trabaja en la ORI y, finalmente, la vinculación de la investigación en los procesos de gestión (Abba, 2015).

Las escuelas normales deben de dotarse de un plan de internacionalización y movilidad académica con el objetivo de armonizar esta ambición con la serie de retos establecidos en sus objetivos estratégicos, de tal forma que se establezcan políticas, objetivos particulares, metas y acciones claras que posibiliten a la comunidad académica la identificación del camino que la institución traza en la materia. Saber qué hacer, sobre qué, dónde y con quién es fundamental para que el proceso de internacionalización sea fructífero y no se limite a una respuesta pasiva a las demandas que provienen del exterior de las instituciones (Larrea y Astur, 2011).

Por otro lado, será importante que las escuelas normales incluyan en los documentos institucionales de planeación (misión, visión, modelo educativo, plan de desarrollo institucional, entre otros) la importancia de la internacionalización y la movilidad como estrategias de desarrollo, y no que simplemente se conciban como proyectos temporales que sean fruto de la iniciativa de una persona o de un periodo de gestión institucional.

Transformaciones al modelo educativo (currículo). Al respecto de la internacionalización del currículo, Knight (2008) y Beelem (2011) definen dos componentes: a) la internacionalización en casa, donde el plan de estudios prepara a los estudiantes para actuar en un mundo global, por lo tanto, las actividades de esta dimensión implican diseñar planes de estudios con enfoque internacional, adaptar los programas de enseñanza, los procesos de aprendizaje, las actividades extracurriculares, el enlace con la cultura local o los grupos étnicos, y las actividades de investigación académica; b) el segundo componente es la internacionalización en el extranjero (también conocida como internacionalización transfronteriza), incluidas todas las formas de educación que sobrepasan las fronteras, es decir, la movilidad de estudiantes y profesores, así como los proyectos de movilidad, programas y servicios educativos.

En este ámbito, la escuela normal requiere de la implementación de una serie de cambios a nivel de la estructura curricular de sus programas, de tal forma que el modelo educativo responda congruentemente a la integración de la dimensión internacional y por ende de la movilidad académica. Algunas estrategias que podemos destacar son las siguientes: 


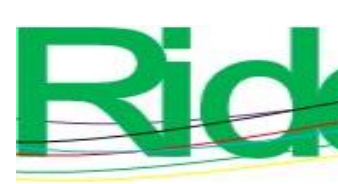

Revista Iberoamericana para la Investigación y el Desarrollo Educativo ISSN 2007 - 7467

internacionalización de agencias y organismos internacionales, y por otro, los programas propios de la institución sobre la cooperación e internacionalización.

En este sentido, será vital para las escuelas normales promover una variedad de convenios y membresías en organismos clave que coadyuven al incremento de estudiantes y docentes participando en estancias académicas a nivel nacional e internacional, ya que muchos de estos gobiernos, organismos, consorcios y agencias ostentan la capacidad de ofrecer becas que, de manera parcial o total, apoyan los objetivos de movilidad que las instituciones de educación superior demandan actualmente.

Independientemente de la diversidad de convenios que tenga una escuela normal para promover su movilidad académica, una aspiración que ha sido poco explotada por las instituciones educativas mexicanas es la procuración de fondos internacionales a través de fundaciones, donantes y asociaciones civiles sin fines de lucro, en donde existe un universo muy amplio de posibilidades para financiar no solo acciones de movilidad académica, sino una gama muy grande de actividades académicas relacionadas con la investigación, la vinculación y el servicio social. Estas universidades o instituciones educativas se han considerado en otros espacios como instituciones "emprendedoras". La universidad emprendedora es un lugar que diversifica sus fondos de forma que no depende esencialmente de los caprichos de los políticos y los burócratas ni tampoco de las corporaciones y tampoco del pago de la matrícula por el estudiantado. El financiamiento en ellas proviene de un conjunto diverso de agencias, exalumnos, intereses de donaciones previas y otras fuentes. Debido a esta diversificación de sus fuentes de financiamiento, las universidades emprendedoras no dependen ni del Estado ni del mercado, sino de la autodeterminación de la universidad de acuerdo con políticas elaboradas por ella (Clark, 2005).

La incursión de las escuelas normales en el mundo de la procuración de fondos es viable en la medida que exista capacitación para el trabajo académico basado en proyectos, además de una transformación de una gestión administrativa y académica más flexible, expedita y con alta visibilidad de resultados. 


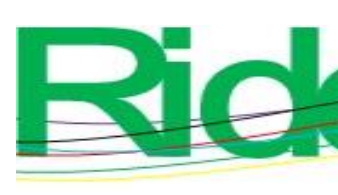

Revista Iberoamericana para la Investigación y el Desarrollo Educativo ISSN 2007-7467

\section{Discusión}

Es evidente que derivado del análisis presentado, la movilidad académica y estudiantil es un tema en la agenda de la investigación educativa en México, especialmente en las escuelas normales mexicanas. El presente artículo pretendió atender, a partir de un análisis documental y descriptivo, la posición que las escuelas normales guardan actualmente en relación con la internacionalización, la movilidad y a los mecanismos en que este subsistema afronta la realidad global de su quehacer formador, el cual sin duda, al menos en México, advierte serios retos que deberán ser atacados desde lo curricular, pero especialmente desde el ámbito de políticas públicas.

La reflexión sobre la necesaria e impostergable adhesión de las escuelas normales a la corriente de la internacionalización abre la puerta a muchas otras perspectivas de corte más pragmático de la academia y gestión institucional: ¿es preciso la aparición de una directriz sobre internacionalización desde la cúpula del sistema educativo nacional para que las escuelas normales adopten acciones en consecuencia?, ¿puede o debe ser responsabilidad de cada institución abordar el reto hacia lo global?, ¿por qué las escuelas normales aún no han establecido los cimientos para que sus comunidades académicas se beneficien de la dimensión internacional a través de la movilidad?

Sin duda, se puede apreciar que las prioridades de cambio estructural tocan los aspectos normativos, financieros, curriculares y organizacionales al interior de las normales que tendrán que ser resueltos en un espectro de mediano y largo plazo; sin embargo, hay otras áreas de oportunidad que pueden ser abordados en la inmediatez y promover en dichas instituciones las condiciones que les permitan construir los programas y proyectos que, en la siguiente década, detonen la participación activa del estudiantado y profesorado en acciones de colaboración académica a nivel nacional e internacional. Entre estos pendientes están, por mencionar algunos, la incorporación de los componentes de interculturalidad e internacionalización en el enfoque de competencias, el establecimiento de convenios y acuerdos de colaboración a partir de experiencias individuales, la promoción del aprendizaje de lenguas extranjeras, la actualización bibliográfica hacia contenidos de corte global o el establecimiento de procedimientos y reglas elementales para las eventuales actividades de movilidad.

Estos cuestionamientos y señalamientos con seguridad detonarán otras brechas de investigación que abonen al abordaje profundo de un tema incipiente para estas instituciones, incentivando no solo a los investigadores pertenecientes a las escuelas normales, sino al creciente 


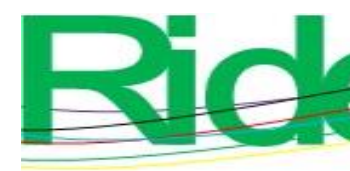

Revista Iberoamericana para la Investigación y el Desarrollo Educativo ISSN $2007-7467$

convenios) aunado a una estrategia que coadyuve al acceso de la comunidad académica a opciones de financiamiento para la movilidad y la internacionalización en general.

Las escuelas normales, como instituciones clave en la formación de profesionales de la educación en México y en Latinoamérica, tienen el gran reto de acoplarse a las tendencias de internacionalización de la educación superior, sin afán de adoptar modelos y estructuras prefabricadas, sino utilizando estos procesos para consolidar su calidad educativa y para abrirse hacia un esquema real de cooperación interinstitucional que fortalezca su esencia formadora.

\section{Referencias}

Abba, M. (2015). Las oficinas de relaciones internacionales en el proceso de internacionalización de la educación superior. Un análisis a través de variables de gestión. Revista Gestão Universitária na América Latina-GUAL, 8(4), 20-37. Doi: https://doi.org/10.5007/1983-4535.2015v8n4p20

Altbach, P. G. (2004). Globalization and the University: Myths and Realities in an Unequal World. In National Education Association, The NEA 2005 Almanac of Higher Education. Washington, D.C: National Education Association.

Altbach, P. G. and Knight, J. (2007). Thcastiellodeardofdíaz e Internationalization of Higher Education: Motivations and Realities. Journal of Studies in International Education, 11(3-4), 290-305. Doi: https://doi.org/10.1177/1028315307303542

Anguas, M. B. M. (2013). Los organismos internacionales y las políticas de formación docente. En Ducoing, P. La escuela normal. Una mirada desde el otro. Universidad Nacional Autónoma de México, Instituto de Investigaciones sobre la Universidad y la Educación, Centro Cultural Universitario, Ciudad Universitaria, Coyoacán, 4510.

Bartell, M. (2003). Internationalization of Universities: A University Culture-Based Framework. Higher Education, 45(1), 43-70.

Beelem, J. (2011). Internationalisation at Home in a Global Perspective: A Critical Survey of the 3rd Global Survey Report of IAU. International Journal of Educational Technology in Higher Education, 8(2), 249-264.

Bok, D. (2006). Our underachieving colleges: A candid look at how much students learn and why they should be learning more. Princeton: Princeton University Press. 


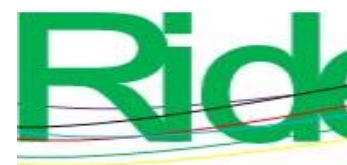
Revista Iberoamericana para la
Investigación y el Desarrollo Educativo
ISSN $2007-7467$

Castiello, S., Cortes, C. y Bustos, M. (2018). ¿Qué sabemos de movilidad estudiantil en México? Logros y retos derivados del reporte Patlani. Recuperado de https://educacion.nexos.com.mx/?p=1086

Clark, B. (2005). The character of the entrepreneurial university. International Higher Education, (38), 2-3. $\quad$ Retrieved from https://ejournals.bc.edu/index.php/ihe/article/view/7456/6652

De Witt, H. (2002). Internationalization of higher education in the United States of America and Europe: A historical, comparative, and conceptual analysis. Westport, CT: Information Age Pub Inc.

Deardorff, D. and Hunter, W. (2006). Educating global ready graduates. International Educator, 72-83.

Díaz Barriga, F. (2005) Enseñanza situada. Vínculo entre la escuela y la vida. México: Mc Graw Hill.

Díaz, A. y Quiroz, R. (2013). La formación integral: una aproximación desde la investigación. Íkala, Revista de Lenguaje y Cultura, 18(3), 17-29. Recuperado de http://www.redalyc.org/pdf/2550/255030038002.pdf

Didou, A. S. (2017) La internacionalización de la educación superior en América Latina: transitar de lo exógeno a lo endógeno. México: UDUAL.

Fernández, J. (2005). Matriz de competencias del docente de educación básica. Revista Iberoamericana de Educación, 36(2), 1-14. Recuperado de https://rieoei.org/historico/investigacion/939Fernandez.PDF

Fresán, M. (2009). Impacto del programa de movilidad académica en la formación integral de los alumnos. Revista de la Educación Superior, 38(151) 141-160. Recuperado de http://publicaciones.anuies.mx/pdfs/revista/Revista151_S4A1ES.pdf

Gacel-Ávila, J. (2005). La internacionalización de la educación superior en América Latina: el caso de México. Cuaderno de Investigación en la Educación, (20), 1-14. Recuperado de https://www.uco.edu.co/ova/OVA\%20Internacionalizacion/ova\%20internacionalizaci\% C3\%B3n/g)\%20Gacel-\%C3\%81vila,\%20J\%20Cuaderno\%20de\%20Investigaci\%C3\%B3n\%20en\%20la\%20Educaci\%C3\%B3n\% 202005.pdf 


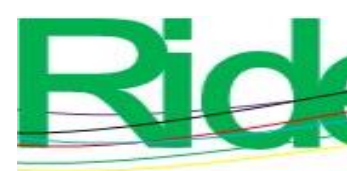

Revista Iberoamericana para la Investigación y el Desarrollo Educativo ISSN 2007-7467

Gacel-Ávila, J. (2009). Marco teórico: modelo de oficinas de relaciones internacionales. En Red SAFIRO II: casos prácticos para la gestión de la internacionalización en universidades (pp. 6-32). Alicante: Oficina de Gestión de Proyectos Internacionales de la Universidad de Alicante.

García, J. (2013). Movilidad estudiantil internacional y cooperación educativa en el nivel superior de educación. Revista Iberoamericana de Educación, (61), 59-76.

González, J. (2007). Internacionalización de la educación superior. Universidades, (33), 3-10, 310. Recuperado de http://ucsj.redalyc.org/articulo.oa?id=37303302

Grasset, C. (2013). Internationalization rationales, obstacles and drivers: A multiple case study of Spanish higher education institutions. University of Minnesota, Minneapolis. Retrieved from https://conservancy.umn.edu/bitstream/handle/11299/150715/GRASSET_umn_0130E_ 13608.pdf?sequence $=1$

Instituto Nacional para la Evaluación de la Educación [INEE] (2016). Panorama educativo de México 2015. Indicadores del sistema educativo nacional. Educación básica y media superior. México. Recuperado de https://www.inee.edu.mx/wpcontent/uploads/2018/12/P1B115.pdf

Knight, J. (2008). Higher Education in Turmoil. The Changing World of Internationalization. Rotterdam: Sense Publishers.

Knight, J. (2012). Student Mobility and Internationalization: Trends and Tribulations. Research in Comparative and International Education, 7(1), 20-33. Doi: https://doi.org/10.2304/rcie.2012.7.1.20

Larrea, M. y Astur, A. (2011). Políticas de internacionalización de la educación superior y cooperación internacional universitaria. Recuperado de http://portales.educacion.gov.ar/spu/files/2011/12/Artículo-Políticas-deinternacionalización

Magaña, M. (2000). Mejoramiento del desempeño docente en la Universidad de Colima a través de la formación de cuerpos académicos (tesis de maestría). Universidad de Colima, Colima.

Mahroum, S. (2000). Highly skilled globetrotters: mapping the international migration of human capital. $R \& D$ Management, 30(1), 23-31. 


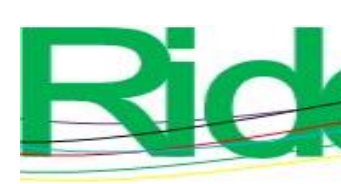

Revista Iberoamericana para la Investigación y el Desarrollo Educativo ISSN $2007-7467$

Martín-Barbero, J. (2003). La globalización en clave cultural: una mirada latinoamericana. Renglones, revista del ITESO, (53), 18-33. Recuperado de https://rei.iteso.mx/bitstream/handle/11117/357/53_02_globalizacion.pdf?sequence=2\& isAllowed $=\mathrm{y}$

Mercado, R. (2000). La incapacidad del plan 1997 de licenciatura en educación primaria. Un estudio sobre el primer semestre (reporte de investigación: temas prioritarios). México, Dirección General de Investigación Educativa SEByN-sep.

Noriega-Chávez, M. (2014) México en la internacionalización. Las escuelas normales. En Navarrete-Cazales, Z. y Navarro-Leal, M. A. (eds.), Internacionalización y educación superior (pp. 301-334). Estados Unidos de América: Palibrio/SOMEC.

Organización para la Cooperación y el Desarrollo Económico [OECD] (2018). Panorama de la educación 2017: indicadores de la OCDE. Madrid: Fundación Santillana. Doi: https://doi.org/10.1787/eag-2017-es

Organización de las Naciones Unidas para la Educación, la Ciencia y la Cultura (UNESCO) (2010). Informe de seguimiento de la educación para todos en el mundo 2010: llegar a los marginados, Francia.

PATLANI (2012). Encuesta nacional de movilidad estudiantil internacional de México. Recuperado de http://www.sincree.sep.gob.mx/work/models/sincree/Resource/archivo_pdf/movilidad. pdf

PATLANI (s. f.). Encuesta mexicana de movilidad internacional estudiantil 2014/2015 y $2015 / 2016$. Recuperado de http://patlani.anuies.mx/archivos/documentos/PATLANI2017_web_optimizado.pdf Rodríguez, H. (2004). Investigación en la escuela normal superior. Revista Colombiana de Educación, (47), 1-14. Recuperado de https://revistas.pedagogica.edu.co/index.php/RCE/article/view/5518/4545

Romo, R. (2006). Políticas públicas, académicos e interdisciplina. Revista de Educación y Desarrollo, (5), 13-20. Recuperado de http://www.cucs.udg.mx/revistas/edu_desarrollo/anteriores/5/005_Romo.pdf

Ruiz, L. (2011). Formación integral: desarrollo intelectual, emocional, social y ético de los estudiantes. Revista Universidad de Sonora, (19), 11-13. Recuperado de http://www.revistauniversidad.uson.mx/revistas/19-19articulo\%204.pdf 


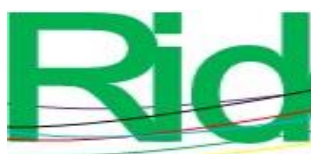

Revista Iberoamericana para la

Saint-Onge, M. (2000) Yo explico, pero ELLOS ... ¿Aprenden?. México: SEP.

Sebastián, J. (2004). Cooperación e internacionalización de las universidades. Buenos Aires: Biblos.

Secretaría de Educación Pública [SEP] (2017). Sistema educativo de los Estados Unidos Mexicanos: principales cifras 2016-2017. Ciudad de México: Autor.

Zaman, K. and Mohsin, A. (2014). Internationalization of Universities: Emerging Trends, Challenges and Opportunities. Journal of Economic Info, (3), 1-9. Doi: https://doi.org/10.31580/jei.v1i1.100 


\begin{tabular}{|c|c|}
\hline Rol de Contribución & Autor (es) \\
\hline Conceptualización & $\begin{array}{l}\text { Román Castro Miranda (Igual) } \\
\text { Héctor Gabriel Rangel Ramírez (igual) }\end{array}$ \\
\hline Metodología & $\begin{array}{l}\text { Román Castro Miranda (Principal) } \\
\text { Héctor Gabriel Rangel Ramírez (Apoyo) }\end{array}$ \\
\hline Software & $\begin{array}{l}\text { Román Castro Miranda (Igual) } \\
\text { Héctor Gabriel Rangel Ramírez (igual) }\end{array}$ \\
\hline Validación & $\begin{array}{l}\text { Román Castro Miranda (Igual) } \\
\text { Héctor Gabriel Rangel Ramírez (igual) }\end{array}$ \\
\hline Análisis Formal & $\begin{array}{l}\text { Román Castro Miranda (Igual) } \\
\text { Héctor Gabriel Rangel Ramírez (igual) }\end{array}$ \\
\hline Investigación & $\begin{array}{l}\text { Román Castro Miranda (Igual) } \\
\text { Héctor Gabriel Rangel Ramírez (igual) }\end{array}$ \\
\hline Recursos & $\begin{array}{l}\text { Román Castro Miranda (Igual) } \\
\text { Héctor Gabriel Rangel Ramírez (igual) }\end{array}$ \\
\hline Curación de datos & $\begin{array}{l}\text { Román Castro Miranda (Igual) } \\
\text { Héctor Gabriel Rangel Ramírez (igual) }\end{array}$ \\
\hline $\begin{array}{l}\text { Escritura - Preparación del } \\
\text { borrador original }\end{array}$ & $\begin{array}{l}\text { Román Castro Miranda (Igual) } \\
\text { Héctor Gabriel Rangel Ramírez (igual) }\end{array}$ \\
\hline $\begin{array}{l}\text { Escritura - Revisión y } \\
\text { edición }\end{array}$ & $\begin{array}{l}\text { Román Castro Miranda (Igual) } \\
\text { Héctor Gabriel Rangel Ramírez (igual) }\end{array}$ \\
\hline Visualización & $\begin{array}{l}\text { Román Castro Miranda (Igual) } \\
\text { Héctor Gabriel Rangel Ramírez (igual) }\end{array}$ \\
\hline Supervisión & $\begin{array}{l}\text { Román Castro Miranda (principal) } \\
\text { Héctor Gabriel Rangel Ramírez (Apoyo) }\end{array}$ \\
\hline $\begin{array}{l}\text { Administración de } \\
\text { Proyectos }\end{array}$ & $\begin{array}{l}\text { Román Castro Miranda (principal) } \\
\text { Héctor Gabriel Rangel Ramírez (Apoyo) }\end{array}$ \\
\hline Adquisición de fondos & $\begin{array}{l}\text { Román Castro Miranda (Apoyo) } \\
\text { Héctor Gabriel Rangel Ramírez (principal) }\end{array}$ \\
\hline
\end{tabular}

